Dermatologische Zeitschrift. 1929;56:I-IV

\title{
Contents, Vol. 56, 1929
}

Inhaltsverzeichnis.

Originalarbeite $\pi$.

Alexander, A., Über die faviforme Degeneration resp. Umwandlung unserer Dermatophyten 225

Brack, Wilh., Untersuchungen an einem Fall von Jodüberempfindlichkeit 26

Danielsen, E., siehe Nag ell, $\mathrm{H}$.

Demuth, Franz, Über ein neues kombiniertes Verfahren zur Behand-

lung des Lupus vulgaris $\quad 125-$

Ebert, M. H., Versuche zur spezifischen Sensibilisierung gegen Tricho-

phytin und Tuberkulin ohne vorausgegangene Infektion 81

Feßler, A., Die bullöse Freibad- und Wiesendermatitis. Bemerkungen

zu E. Urbachs „Physikalische Allergie der Haut” 260

Fonrobert, H., siehe Schmidt-La Baume, F.

Frieboes, W., Beiträge zur Anatomie undBiologie der Haut. XII. Chorda-,

Knorpel-, Epidermiszellen.- Epidermisfasersystem ein System oberer

Ordnung. - Metaplasie von Epidermis- und Haut-Ca-Zellen. - Epi-

dermisbildung beiWundheilung und normale Epidermisregeneration. -

Entstehung der Hautkrebse 305-

- Beitrag zum Bau der Blutgefäßendothelwand

Grigorjew, P., und K. Jarischewa, Zur Frage der spezifischen

Aortitis bei experimenteller Kaninchensyphilis 395

Herzberg, K., siehe Manteufel, P.

Hoffmann, Erich, und EudolfStrempel, Gehirninfektionundmeta-

statische Erkrankung von Hoden und Skrotalhaut (Pseudoprimäraffekt)

nach intrazisternaler Impfung von Kaninchen mit Syphilis .... 1 Jarischewa, K., siehe Grigorjew,

P. Kadisch, E., Über den Einfluß der Züchtungstemperatur auf das An-

gehen und die Größe der Kolonien pathogener Hautpilze 406

Kaufmann, C, Zur histologischen Diagnostik luischer Plazenten (ein-

schließlich des Spirochaetennachweises) $\quad 346$

Loeb, H., Salvarsan bei Erkrankungen der Harnwege 119.

v. Mallinckrodt-Haupt, Asta, Milbenerkrankungen beim Menschen 98

- $\quad$ Katzenkabies beim Menschen232

Manteufel, P., und K. Herzberg, Vírusnachweis durch Drüsenverimpfung beim lebenden kongenital syphilitischen Säugling 340»

Marchionini, Alfred, Säurebehandlung intertriginöserEpidermophytien 248 Migge, Magda, Über einen mit Follikulin geheilten Fall von Impetigo

herpetifo $\pi \mathrm{ms}$ bei einer nicht graviden Frau 110-

Nagell, H., und E. Danielsen, Zur Biologie des Gonokokkus ... 16 Schilling, Viktor, Einige

Bemerkungen zum Wesen und zur prak- 
tischen Bedeutung der Guttadiaphotmethode 261

Schmidt-La Baume, F., und H. Fonrobert, Über ballistische elastometrische Messungen am Gelatineschichtmodell im Vergleich zur menschlichen Haut 95

IV Inhaltsverzeichnis.

S $\theta$ ite

Schreus, H.Th., und Elisabeth Willms, Über den Ausfall derBoltzreaktion in den verschiedenen Stadien der Lues 8

Strempel, Rudolf, siehe Hoffmann, Erich.

Szentkirályi, S. v., Zur Frage der Wirkungsweise der Salvarsanpräparate 128

Urbach, Erich, Physikalische Allergie der Haut. Bemerkungen zur

Arbeit von Oppenheim und Feßler: „Die bullöse Freibad- und Wiesen-

dermatitis" in Bd. 55 S. 191 dieser Zeitschrift 256

Vásárhelyi, J., Zur Frage der Beeiaflußbarkeit der Thalliumwirkung

durch Extrakte endokriner Drüsen, mit· besonderer Berücksichtigung

der Thymus 412

Waldeyer, L., Erfahrungen über Myosalvarsan bei Säuglingen . . 355

Willms, Elisabeth, siehe Schreus, H. Th.

Wohlstein, Emanuel, Ein Fall von Nekrophorus-Infektion der Haut

beim Menschen 415

Domenico Majocchi $† 79$

Gesellschaftsberichte.

Berliner Dermatologische Gesellschaft. Sitzung vom 8.1. 1929

Sitzung vom 12. II. $1929 \quad 135$

Sitzung vom 12. III. $1929 \quad$. $\quad 266$

Sitzung vom 14. V. $1929 \quad 360$

Sitzung vom 11. VI. $1929 \quad 417$

Kölner Dermatologische Gresellschaft. Sitzungen vom 25.1, und 22. II. 1929

52. Tagung der Vereinigung südwestdeutscher Dermatologen zu Frank

furt a. M. am 2. und 3. III. $1929 \quad 168$

Tagung der Vereinigung rheinisch-westfälischer Dermatologen in Düssel-

dorf am 20.1. $1929 \quad 52$

Deutsche Dermatologische Gesellschaft in der Tschechoslowakischen

Republik. Sitzungen vom 23. und 24. II. 1929153

Société Française de Dermatologie et de Syphiligraphie. Sitzungen vom

8. III. und 19. IV. $1928 \quad 59$

- $\quad$ Sitzungen vom 14. VI. und 12. VII. 1928184

Royal Society of Medicine, Section of Dermatology. Sitzungen vom

18. X. und 15. XI. $1928 \quad 63$

Britische Gesellschaft für Dermatologie und Syphilis. 8. Kongreß vom

12. und 13. VII. 1928188

Gesellschaft für Haut- und Geschlechtskrankheiten in Kiew. Sitzungen ,

vom 2. und 19. XII. $1928 \quad 189$

Periodische Literatur. Zusammengestellt von Dr. Arthur Alexander-

Charlottenburg $\quad 66,191,278,369,434$

Buchbesprechungen 75, 219, 303, 444 
Personalien und Tagesnachrichten $\quad 80,223,304,380,381,446$

50 Jahre Polikli $\pi$ ik far Haut- und Geschlechtskrankheiten in Bonn .... 80

Sachregister 448

Namenregister 457 